Mojca Leskovec

UDK 811.112.2'243:811.111

Philosophische Fakultät, Universität Ljubljana

Slowenien

mojca.leskovec@ff.uni-lj.si
DOI: 10.4312/vestnik.11.191-199

\title{
FALSCHE FREUNDSCHAFT ODER INTERFERENZFEHLER SLOWENISCHER DEUTSCHLERNENDER AUS DEM ENGLISCHEN
}

\section{EINFÜHRUNG}

Spätestens seit dem Erscheinen des Gemeinsamen Europäischen Referenzrahmens für Sprachen (weiter GeR) und verstärkt insbesondere nach dem Erscheinen des Referenzrahmens für plurale Ansätze zu Sprachen und Kulturen (weiter REPA) gewinnt die Mehrsprachigkeit im Fremdsprachenunterricht an Bedeutung. Diese ist laut dem GeR (2001) von der Vielsprachigkeit als Kenntnis einer Anzahl von Sprachen oder Koexistenz verschiedener Sprachen in einer bestimmten Gesellschaft zu unterscheiden:

Mehrsprachigkeit /.../ betont die Tatsache, dass sich die Spracherfahrung eines Menschen in seinen kulturellen Kontexten erweitert, von der Sprache im Elternhaus über die Sprache der ganzen Gesellschaft bis zu den Sprachen anderer Völker /.../Diese Sprachen und Kulturen werden aber nicht in strikt voneinander getrennten mentalen Bereichen gespeichert, sondern bilden vielmehr gemeinsam eine kommunikative Kompetenz, zu der alle Sprachkenntnisse und Spracherfahrungen beitragen und in der die Sprachen miteinander in Beziehung stehen und interagieren.

Diese Erkenntnis manifestiert sich u. a. in der Mehrsprachigkeitsorientierung als einem der didaktisch-methodischen Prinzipien der Postmethodenära. Es ist demnach nicht mehr verboten, wie dies in der Geschichte der fremdsprachlichen Didaktik mehrmals der Fall war, die Erstsprache sowie andere Sprachen in den Unterricht der Zielsprache mit einzubeziehen. Vielmehr ist es empfehlenswert, sich das Kennen und Können von anderen Sprachen dabei zunutze zu machen.

Unbestreitbar beeinflussen die bereits gelernten Sprachen das Erlernen einer weiteren Sprache; allerdings nicht immer nur positiv. Dieser Beitrag setzt sich deshalb zum Ziel, am Beispiel der slowenischen Deutschlernenden der Sekundarstufe II den negativen Transfer aus dem Englischen als ihrer mehrheitlich ersten Fremdsprache zu schildern und 
zu erörtern, wie man diesen in den Unterricht einbeziehen soll, damit der Lernprozess effizienter und ökonomischer erfolgt. Dargestellt werden Interferenzerscheinungen, die die Autorin dieses Beitrags in ihrer Unterrichtspraxis am Gymnasium jahrelang gesammelt hat. Dennoch stellen diese lediglich einen geringen Teil dieses wichtigen, weiterer Forschung bedürftigen Gebietes.

\section{MEHRSPRACHIGKEITSORIENTIERUNG IM FREMDSPRACHENUNTERRICHT}

Im Sinne der Mehrsprachigkeit kann man nach dem GeR (Europarat 2001) ,auf die Kenntnis mehrerer Sprachen zurückgreifen, um den Sinn eines geschriebenen oder gesprochenen Textes zu verstehen, der in einer eigentlich, unbekannten' Sprache verfasst wurde; dabei erkennt man zum Beispiel Wörter aus einem Vorrat an Internationalismen, die hier nur in neuer Gestalt auftreten“. Laut dem REPA (Candelier et al. 2012) wird durch plurale Ansätze sowohl die Entwicklung der Fertigkeit, „die in einer Sprache verfügbaren Kenntnisse und Fertigkeiten für Handlungen des Sprachverstehens oder der Sprachproduktion in einer anderen Sprache nutzen /zu/ können“, gefördert als auch das Wissen, „dass zwischen Sprachen oder sprachlichen Varietäten Ähnlichkeiten und Unterschiede bestehen".

Wie einem der zentralen Leitgedanken von Mehrsprachigkeitsdidaktik zu entnehmen ist, erreicht man gerade aufgrund von bereits vorhandenen Sprachkenntnissen einen schnelleren Lernfortschritt in einer neuen Fremdsprache; zwar können diese das Lernen einer weiteren Sprache sowohl positiv als auch negativ beeinflussen, doch wie Pilypaityté (2013: 141, 144) aufgrund neuerer wissenschaftlicher Erkenntnisse feststellt, ist der positive Transfer viel umfangreicher als der negative. Auch Hu (2016: 13) betont, dass im Sprachenunterricht die Kultur der Mehrsprachigkeit gefördert werden soll, denn „Sprachvergleiche semantischer, morphologischer, lexikalischer und syntaktischer Art können Spezifika der unterschiedlichen Sprachen erhellen, ,Fehler` und faux amis erklären sowie spezifische Blickrichtungen auf die Welt verdeutlichen“. Pilypaityté (2012: 19) fasst die bei verschiedenen Ansätzen der Mehrsprachigkeitsdidaktik zu findenden Prinzipien mit dem Dreischritt Bewusstmachen - Vergleichen - Anknüpfen zusammen.

Die Sprachenkonstellation Deutsch nach Englisch ist nicht nur für Slowenien charakteristisch, sondern auch in anderen Ländern der Welt durchaus häufig. Dies bedeutet, dass die Lernenden in den Deutschunterricht die Erfahrungen mit dem Lernen von mindestens zwei anderen Sprachen, d. i. der Erstsprache bzw. Muttersprache sowie der ersten Fremdsprache, mitbringen. Die Vorerfahrungen der Lernenden ermöglichen es nach Hufeisen (2003: 9), dass der Unterricht der zweiten Fremdsprache auf einer höheren Stufe beginnt, die Progression steiler angelegt ist und die Inhalte anspruchsvoller sind. Diese Situation bedarf somit der Tertiärsprachendidaktik. Dabei geht es allerdings nicht 
zunächst um die Frage nach den Unterschieden, sondern nach dem (positiven) Transfer: dieser bezieht sich zum einen auf die Erweiterung des Sprachbesitzes und zum anderen des Sprachlernbewusstseins (Neuner 2003: 24-26). Neuner (ebd.: 27-32) führt fünf Prinzipien der Tertiärsprachendidaktik an, die den jeweiligen regionalen und lerngruppenspezifischen Gegebenheiten angepasst werden sollen: kognitives Lernen - Entfaltung von Sprachbewusstheit und Sprachlernbewusstheit, Verstehen als Grundlage und Ausgangspunkt des Lernens, Inhaltsorientierung, Textorientierung und Ökonomisierung des Lernprozesses. Insbesondere im Tertiärsprachenunterricht dürfen und sollen demnach bewusstmachende Unterrichtsverfahren angewendet werden.

\section{3}

\section{TRANSFERERSCHEINUNGEN IM LERNPROZESS}

Nach Neveling (2016: 118) stellen Lernende bei der Autosemantisierung „eine Hypothese über eine Wortbedeutung auf, indem sie einen Transfer auf der Basis vier möglicher Wissensquellen vornehmen": formähnliche Wörter aus anderen Sprachen, Kenntnisse des Zielsprachenwortes und der Wortbildungsregeln, das allgemeine Weltwissen sowie der Kontext. Laut Marx (2016: 296) scheint die Lexik anfälliger für den L2-Transfer zu sein, während bei syntaktischen Strukturen oder pragmatischem Handeln häufiger von L1-Transfererscheinungen berichtet wird.

Neuner et al. (2009: 70) weisen darauf hin, dass der Vergleich und das Einordnen neuer grammatikalischer Phänomene in die bereits vorhandenen Strukturen bei Lernenden früher oder später als eine Art „stummer Prozess“ stattfinden. „Wenn also Interferenz-Fehlerquellen nicht rechtzeitig erklärt und geklärt werden, ist das Produzieren solcher Fehler faktisch programmiert“, fügen sie hinzu (ebd.). Marx (2016: 297) berichtet, dass diejenigen L3-Lernenden, ,die im Rahmen des L3-Unterrichts explizit auf ihre anderen Sprachen und ihre Sprachlernerfahrungen hingewiesen werden, Sprachenvergleiche gezielt üben und somit die Systematik von Sprachen reflektieren", tatsächlich erfolgreicher sind. Krevs Birk (2014: 361) konstatiert, dass zur sprachlichen und somit zur kommunikativen und kulturellen Kompetenz auch das sprachliche Wissen über Unterschiede in Bedeutungsstrukturen einzelner Lexempaare gehört, ,denn aufgrund der Ähnlichkeit der Formative können bestimmte Lexempaare zu falschen Freunden werden“ (Krevs Birk 2014: 361).

Den negativen Transfer von einer anderen Sprache auf die Zielsprache bezeichnet man in der Sprachwissenschaft als Interferenz. Bußmann (2008: 301) definiert Interferenz als Beeinflussung eines Sprachsystems durch ein anderes im Individuum oder in der Sprachgemeinschaft. Dabei kann es sich um die lexikalische, phonologische, morphologische, orthographische, semantische oder syntaktische Ebene handeln. Zu den Interferenz-Fehlern auf der lexikosemantischen Ebene können sog. falsche Freunde führen, nach Bußmann (ebd.: 189) „Paare von Wörtern aus verschiedenen Sprachen, die trotz formaler Ähnlichkeiten verschiedene Bedeutungen haben“. 
Allen in die Beobachtung herangezogenen mehr als 400 slowenischen Gymnasiasten und Gymnasiastinnen war gemeinsam, dass ihre Erstsprache Slowenisch war und dass sie institutionell Englisch als erste und Deutsch als zweite Fremdsprache lernten. Nicht ausschließen ließ sich dabei allerdings die Möglichkeit, dass sie informell noch andere Sprachen gekannt oder gelernt hatten. Es ließen sich in der Unterrichtspraxis insbesondere semantische Interferenzfehler aus dem Englischen feststellen, in einem kleineren Umfang allerdings auch morphologische sowie orthographische.

Auf der morphologischen Ebene wurde das Partizip II, wie dies im Englischen üblich ist, beispielsweise mit der Endung -ed statt -et gebildet:"befreunded statt befreundet. Ferner ließ sich beim Superlativ in attributiver Stellung des Adjektivs das Suffix -est statt -(s)t wie im Beispiel ${ }^{*} a m$ größest statt am größten finden; zwar ist auch im Deutschen dieses Suffix möglich, jedoch bei einsilbigen oder endbetonten Adjektiven auf $-d,-t,-s,-\beta$, $-x,-z$, wobei auch diese in attributiver Stellung der entsprechenden Endung -en bedürfen. Eine Ähnlichkeit mit der englischen Entsprechung the biggest lässt sich kaum übersehen. Ähnlich stammte das Suffix -er beim folgenden Substantiv wohl von seiner englischen Entsprechung: *der Philosopher statt der Philosoph.

Auf der orthographischen Ebene war die häufigste Interferenzerscheinung aus dem Englischen das allgemeine Großschreiben des Personalpronomens für die erste Person Singular, also Ich statt ich wie im Englischen $I$. Es ließen sich in Fällen von formativisch ähnlichen Lexemen ferner folgende orthographische Fehler beobachten: ${ }^{*}$ under statt unter, ${ }^{*}$ drinken statt trinken, ${ }^{*}$ der Garden statt der Garten.

Wie bereits hervorgehoben, waren die semantischen Interferenzen jedoch am häufigsten. Neben dem in diesem Kontext in unterschiedlichen Quellen schon mehrmals angeführten Wortpaar dt. bekommen - engl. to become, dt. werden, waren bei slowenischen Deutschlernenden der Sekundarstufe II noch folgende (potenzielle) falsche Freunde zu finden: dt. wer - engl. where, dt. wo, dt. also - engl. also, dt. auch, dt. vor - engl. for, dt. für, dt. bei - engl. by, dt. von, dt. lösen - engl. to lose, dt. verlieren, dt. reden - engl. to read, dt. lesen, dt. laden - engl. to lead, dt. führen, dt. man - engl. man, dt. der Mann, dt. das Gift - engl. gift, dt. das Geschenk. In allen angeführten Fällen geht es um formähnliche Wörter, die zur Annahme führen, dass sie sich auch semantisch entsprechen, was ansonsten eigentlich nicht selten der Fall ist. Bei dem ebenso oft vorkommenden Wortpaar dt. der Student - engl. student, dt. der Schüler, geht es beispielsweise tatsächlich um Teiläquivalenz.

Aus dem in den Kapiteln 2 und 3 dargestellten Forschungsstand lässt sich die Schlussfolgerung ziehen, dass man sich im Tertiärsprachenunterricht der Mehrsprachigkeit sowie 
der Kognitivierung bedienen soll. Ausgegangen werden soll zwar von Gemeinsamkeiten und Ähnlichkeiten zwischen den Lernenden bekannten Sprachen, doch auch Unterschiede dürfen nicht zu lange beiseitegelassen werden. Vielmehr soll man den Vergleich aufbauen, indem man rechtzeitig auch Unterschiede zwischen diesen Sprachen anspricht und sie bewusst macht. Das Feststellen potenzieller Interferenzquellen seitens der Lehrkraft und das Erstellen einer entsprechenden Liste ist dabei nur der erste, aber nicht ausreichende Schritt.

Es ist in erster Linie notwendig, die eigene Lerngruppe genau kennen zu lernen: welche Erstsprachen sind darin vorhanden, inwieweit unterscheiden sich diese von der Sprache der Umgebung, welche anderen Sprachen wurden gelernt, auf welcher Niveaustufe sind deren Kenntnisse, wie sind die bisherigen Erfahrungen mit dem Sprachenlernen bzw. -unterricht u. a. m. Eine diesbezügliche in den ersten gemeinsamen Deutschstunden gezielt durchgeführte Befragung kann sich dabei als äußerst nützlich erweisen. Nicht nur die Möglichkeiten des positiven Transfers von diesen Sprachen auf die Zielsprache lassen sich somit leichter feststellen, sondern auch die Quellen von (potenziellen) Interferenzfehlern.

Im Sinne der Erfolgsorientierung und Motivierung beginnen die ersten Unterrichtsstunden Deutsch als zweite Fremdsprache tatsächlich häufig mit Einführung von Internationalismen. Dadurch sind die Lernenden schon zu Beginn in der Lage, ihre bereits vorhandenen Erfahrungen und Kenntnisse zu aktivieren. Nach den Gemeinsamkeiten kommen demnächst allerdings Unterschiede vor: im Deutschen schreibt man Substantive anders als im Englischen groß. Und sobald die Lernenden etwa die erste Aussage über sich selbst machen und dabei das Personalpronomen für die erste Person Singular eingeführt wird, soll darauf hingewiesen werden, dass dieses anders als im Englischen im Deutschen klein geschrieben wird. Auch bei Lexik ist es im Allgemeinen empfehlenswert, die potenziellen falschen Freunde im Voraus zu klären. Dies kann durch eine einfache Frage, etwa Wie heißt denn to read auf Deutsch?, und die anschließende Diskussion im Sinne von ... lesen, also anders als im Englischen geschehen.

Die Lerngruppen und die Lernenden individuell unterscheiden sich voneinander $\mathrm{u}$. a. in dem Grad des bereits entwickelten Sprachbewusstseins sowie des Interesses am Sprachenlernen. Während einige sich selbst des Sprachvergleiches bedienen, müssen andere erst dazu erzogen werden. Es liegt also an der Lehrkraft, dieses enorme Potenzial zu pflegen bzw. zu aktivieren. Behilflich können der Lehrkraft dabei etwa motivierende Themen und Texte sein, die Letzteren möglicherweise parallel in beiden Sprachen, so dass sie einen automatisch zum Vergleichen ansprechen.

Wie einleitend angedeutet, wird das Lernen einer (neuen) Sprache von allen bis zu diesem Zeitpunkt erlernten und gelernten Sprachen beeinflusst. Um dabei ökonomischer 
vorzugehen und eine höhere Effizienz zu erzielen, soll man sich allerdings nicht lediglich die Ähnlichkeiten zwischen Sprachen und ihre Gemeinsamkeiten zunutze machen, sondern sich auch der Unterschiede bewusst werden. Dieser Beitrag setzte sich deshalb zum Ziel, am Beispiel der slowenischen Deutschlernenden der Sekundarstufe II die Interferenzerscheinungen aus dem Englischen als ihrer mehrheitlich ersten Fremdsprache zu schildern. Es wurde in Bezug auf Theorie und Praxis ebenso erörtert, wie man sie in den Unterricht einbeziehen soll.

Hiermit wurde allerdings lediglich ein geringer Teil dieses breiten Gebietes dargestellt. Insbesondere für die weltweit durchaus häufige Sprachenkonstellation Deutsch nach Englisch stehen inzwischen zahlreiche Lehrwerke zur Verfügung, die den Lehrenden eine diesbezügliche Unterstützung anbieten. An dieser Stelle sei jedoch erneut hervorgehoben, dass nicht nur das Englische als die erste Fremdsprache, sondern auch die jeweiligen mehr oder wenig verwandten Erstsprachen der Lernenden das Lernen des Deutschen als ihrer zweiten Fremdsprache beeinflussen (s. dazu auch Leskovec 2018 und Muster 2000). Dieses nicht unwichtige Gebiet wird - wohl auch aufgrund äußerst heterogener Zielgruppen der Lehrwerke - im Allgemeinen allerdings den Lehrkräften überlassen, die nach dem lange geltenden Prinzip der Einsprachigkeit nun dafür auch selber im Rahmen von Seminaren und Fortbildungen entsprechend sensibilisiert werden sollten.

\section{BIBLIOGRAPHIE}

BUßMANN, Hadumod (2008) Lexikon der Sprachwissenschaft. 4., durchgesehene und bibliographisch ergänzte Auflage. Stuttgart: Alfred Kröner Verlag.

CANDELIER, Michel/Antoinette CAMILLERI GRIMA/Véronique CASTELLOTTI / Jean-François DE PIETRO/Ildikó LŐRINCZ/Franz-Joseph MEIßNER/Muriel MOLINIÉ/Artur NOGUEROL/Anna SCHRÖDER-SURA (2012) Referenzrahmen für plurale Ansätze zu Sprachen und Kulturen. 19. Juli 2019. https://carap.ecml.at/Accueil/tabid/3577/language/de-DE/Default.aspx.

EUROPARAT (Hrsg.) (2001) Gemeinsamer Europäischer Referenzrahmen für Sprachen: lernen, lehren, beurteilen. 19. Juli 2019. https://www.goethe.de/Z/50/ commeuro/i0.htm.

HUFEISEN, Britta (2003) Kurze Einführung in die linguistische Basis. B. Hufeisen und G. Neuner (Hrsg.), Mehrsprachigkeitskonzept - Tertiärsprachenlernen - Deutsch nach Englisch. Strasbourg: Europarat, 7-11. 28. Juli 2019. https://www.ecml.at/ Portals/1/documents/ECML-resources/TER-GE.pdf?ver=2018-04-17-153342-267.

KREVS BIRK, Uršula (2014) Deutsche und slowenische sprachliche Beziehungen und interkulturelle Linguistik: Beispiel Internationalismen. M. Lah (Hrsg.), Skupni evropski jezikovni okvir - navzkrižni pogledi $=$ Cadre européen commun de référence 
pour les langues - regards croisés. Ljubljana: Znanstvena založba Filozofske fakultete, 353-363.

LESKOVEC, Mojca (2018) Vom Slowenischen als Erst- zum Deutschen als Fremdsprache: Wege zur Entwicklung grammatischer und kommunikativer Kompetenz. Vestnik za tuje jezike, 1, 187-197.

MARX, Nicole (2016) Lernen von zweiten und weiteren Fremdsprachen im Sekundarschulalter. E. Burwitz-Melzer, G. Mehlhorn, C. Riemer, K.-R. Bausch und H.-J. Krumm (Hrsg.), Handbuch Fremdsprachenunterricht. 6., völlig überarbeitete und erweiterte Auflage. Tübingen: A. Francke Verlag, 295-300.

MUSTER, Nanika (2000) Napake slovenskih učencev pri učenju nemščine. Ljubljana: Zavod Republike Slovenije za šolstvo.

NEUNER, Gerhard (2003) Mehrsprachigkeitskonzept und Tertiärsprachendidaktik. B. Hufeisen und G. Neuner (Hrsg.), Mehrsprachigkeitskonzept - Tertiärsprachenlernen - Deutsch nach Englisch. Strasbourg: Europarat, 13-34. 28. Juli 2019. https://www.ecml.at/Portals/1/documents/ECML-resources/TER-GE.pdf?ver=2018-04-17-153342-267.

NEUNER, Gerhard/Britta HUFEISEN/Anta KURSIŠA/Nicole MARX/Ute KOITHAN/ Sabine ERLENWEIN (2009) Deutsch als zweite Fremdsprache: Fernstudieneinheit 26. Berlin [etc.]: Langenscheidt.

NEVELING, Christiane (2016) Verfügen über sprachliche Mittel: Wortschatz. E. Burwitz-Melzer, G. Mehlhorn, C. Riemer, K.-R. Bausch und H.-J. Krumm (Hrsg.), Handbuch Fremdsprachenunterricht. 6., völlig überarbeitete und erweiterte Auflage. Tübingen: A. Francke Verlag, 116-121.

PILYPAITYTÉ, Lina (2013) Wie helfen andere Sprachen beim Deutschlernen? S. Ballweg, S. Drumm, B. Hufeisen, J. Klippel und L. Pilypaityté, Deutsch lehren lernen 2: Wie lernt man die Fremdsprache Deutsch? München: Goethe-Institut, Klett-Langenscheidt, $132-170$.

PILYPAITYTÉ, Lina (2012) Argumente für die Mehrsprachigkeit: Sprachenpolitische Forderungen und ihre Umsetzung im schulischen Kontext. Ljubljana: Goethe-Institut, Ministrstvo za izobraževanje, znanost, kulturo in šport.

POVZETEK

\section{Lažno prijateljstvo ali interference slovenskih učencev in učenk nemščine iz angleščine}

V Skupnem evropskem jezikovnem okviru predstavljeno izhodišče, da obstaja le ena sporazumevalna zmožnost, h kateri prispevajo celotno znanje in izkušnje z jezikom ter v kateri se vsi jeziki povezujejo in medsebojno delujejo, se kaže med drugim v raznojezični usmerjenosti kot enem izmed didaktično-metodičnih načel postmetodske dobe. Skladno z njim je pri učenju novega jezika 
priporočljivo izkoristiti poznavanje in znanje drugih jezikov. Jeziki, ki smo se jih učili, pa lahko na učenje nadaljnjega jezika vplivajo ne le pozitivno, temveč vendarle tudi negativno. Ta prispevek si zato postavlja cilj, na primeru slovenskih učencev in učenk nemščine kot drugega tujega jezika v gimnaziji prikazati interference iz angleščine kot njihovega večinskega prvega tujega jezika in nato obravnavati način vključevanja interferenc $\mathrm{v}$ pouk, da bi bil proces učenja tako lahko učinkovitejši in gospodarnejši. Predstavljeni so pojavi interferenc, ki jih je avtorica prispevka zbirala pri svojem večletnem pedagoškem delu v gimnazijskem programu. Pri tem so se najpogosteje pojavljale interference na semantični ravni, v manjšem obsegu pa tudi interference na morfološki in pravopisni ravni.

Ključne besede: nemščina kot drugi tuji jezik, sporazumevalna zmožnost, raznojezičnost, transfer, interferenca

\section{ABSTRACT}

\section{False friends or interferences of English with Slovene learners of German}

The discovery, postulated in the Common European Framework of Reference for Languages, that there is only one communicative competence, to which all knowledge and experience of language contribute and in which languages interrelate and interact, manifests itself amongst other things in the plurilingual orientation as one of the many didactic-methodological principles of the post-method era. According to this, it is recommended to take advantage of the knowledge of other languages when learning a new language. The languages one has learned, however, can affect the learning of a further language in a positive as well as a negative way. The aim of this article is to show the interferences of English as the first foreign language on the example of Slovene learners of German as a second foreign language in a secondary school, and to discuss how to integrate them in the language classroom so that the learning process would be more effective and efficient. The article presents interferences that the author collected in her years teaching at secondary school. Most of them are semantic, although morphological and orthographic interferences could also be found.

Keywords: German as a second foreign language, communicative competence, plurilingualism, transfer, interference

\section{ZUSAMMENFASSUNG}

Die im Gemeinsamen Europäischen Referenzrahmen für Sprachen postulierte Erkenntnis, dass es nur eine kommunikative Kompetenz gibt, zu der alle Sprachkenntnisse und Spracherfahrungen beitragen und in der die Sprachen miteinander in Beziehung stehen und interagieren, manifestiert 
sich u. a. in der Mehrsprachigkeitsorientierung als einem der didaktisch-methodischen Prinzipien der Postmethodenära. Es ist demnach empfehlenswert, sich das Kennen und Können von anderen Sprachen beim Lernen einer neuen Sprache zunutze zu machen. Die bereits gelernten Sprachen können das Erlernen einer weiteren Sprache allerdings nicht nur positiv, sondern auch negativ beeinflussen. Dieser Beitrag setzt sich deshalb zum Ziel, am Beispiel der slowenischen Deutschlernenden der Sekundarstufe II die Interferenzerscheinungen aus dem Englischen als ihrer mehrheitlich ersten Fremdsprache zu schildern und zu erörtern, wie man sie in den Unterricht einbeziehen soll, damit der Lernprozess effizienter und ökonomischer erfolgt. Dargestellt werden Interferenzerscheinungen, die die Autorin dieses Beitrags in ihrer Unterrichtspraxis am Gymnasium jahrelang gesammelt hat. Es ließen sich dabei insbesondere semantische Interferenzfehler aus dem Englischen feststellen, in einem kleineren Umfang allerdings auch morphologische sowie orthographische.

Schlüsselwörter: Deutsch als zweite Fremdsprache, kommunikative Kompetenz, Mehrsprachigkeit, Transfer, Interferenz 